\section{Interações sociais no Facebook: um estudo sobre a construção dos processos de capital social* $^{*}$}

\section{Social interactions on Facebook: a study on the processes construction of social capital}

Arthur Maia Vargas ${ }^{1}$

Regina Celia Xavier dos Santos ${ }^{2}$
Recebido em: 27/04/2015.

Aprovado em: 30/05/2015.

1 Graduado em Publicidade e Propaganda pelo Centro Universitário de Brasília. Certificado em Marketing pela Wharton School of the University of Pennsylvania. Desenvolve trabalhos na área da Comunicação com ênfase em mídias sociais, comportamento do consumidor e web design. Endereço: SHIN QL 15 Conjunto 7 Casa 19 - Lago Norte. CEP: 71535-275 - Brasília - DF. E-mail: arthur.mvargas@gmail.com. cel: (61) 9844-2125.

2 Graduada em Estatística pela Universidade Estadual de Campinas. Mestre em Matemática Aplicada pela Universidade Estadual de Campinas. Professora do Centro Universitário de Brasília. Experiência na área de Pesquisa de Mercado e Ciência da Informação, com ênfase em Comportamento do Consumidor. Endereço: Centro Universitário de Brasília, Faculdade de Ciências Sociais Aplicada - Depar-tamento de Comunicação Social. SEPN 707/907 - Campus do Uniceub - Bloco 12 Asa Norte 70790-075 - Brasília - DF. Homepage: www.uniceub.com.br.

\section{Resumo}

Este estudo teve por objetivo geral analisar a formação de capital social do tipo bridging, no contexto virtual do Facebook e no contexto social das interações face a face. Trata-se de uma pesquisa de campo, do tipo quantitati-va e de caráter exploratório. Participaram do estudo 220 estudantes de um centro universitário do Distrito Federal. Foi utilizada versão traduzida dos questionários "General Bridging Social Capital" e "Facebook Bridging Social Capital". A análise dos dados foi feita com o Statistical Package for Social Sciences (SPSS), tendo sido realizadas análises descritivas e fatoriais. Os resultados obtidos com a tradução e validação dos questionários para a versão em Português, primeiro objetivo específico da pesquisa, mostraram-se bastante semelhantes aos resultados encon-trados na versão original, sinalizando que o instrumento parece adequado para se investigar as relações que ocorrem na formação de capital social do tipo bridging dentro e fora do Facebook. O resultado encontrado nas comparações, segundo objetivo específico da pesquisa, evidenciou que, na amostra empregada, a criação do capital social do ti-po bridging não é fortemente afetada pela utilização da rede Facebook, uma vez que não houve diferenças signifi-cativas quando comparando a criação desse tipo de capital social dentro e fora do Facebook.

Palavras-chave: Capital social. Redes sociais. Facebook.

\begin{abstract}
This study aimed to analyse the formation of bridging social capital within and out of the Facebook network. It is a field study, quantitative and characterised as exploratory. A sample of 220 undergraduates was taken from an university in Distrito Federal. A translated version was used of the questionnaires "General Bridging Social Capital" and "Facebook Bridging Social Capital". There were made descriptive and factorial analysis of the data using the Statistical Package for Social Sciences (SPSS). The results obtained with the translation and validation of the questionnaires to the Portuguese version, which was the first specific goal of the research, showed great resem-blance with the results encountered in the original version, signalising that the instrument seems adequate to investi-gate the relationships that occur on the formation of bridging social capital within and out of the Facebook network. The result obtained from the comparisons, which stands as the second specific goal of the research, evidenced that, taking the sample in question, the formation of bridging social capital is not strongly affected by the use of the Fa-cebook network, bearing that there were no significant differences when comparing the formation of that type of social capital within and out of the Facebook.
\end{abstract}

Keywords: Social capital. Social network. Facebook. 


\section{Introdução}

Existem hoje na literatura nacional e estrangeira muitos estudos relacionados às redes sociais, particularmente no que diz respeito ao fenômeno Facebook. Estudiosos de diferentes áreas do conhecimento como, por exemplo, Psicologia, Informática, Marketing e Publicidade estão procurando entender as dinâmicas de comunicação dessas redes sociais. No Brasil, Primo (2007) estudou as interações de blogueiros nas redes sociais; Barros (2010) investigou as inovações da comunicação entre empresas e consumidores nas mídias digitais; Cruz et al (2012) estudaram o boicote de consumidores nas redes sociais virtuais; Ciribeli e Paiva (2011) pesquisaram a utilização das redes e mídias por brasileiros e sua influência na vida dos usuários. Na literatura estrangeira, Karjaluoto e Leppäniemi (2013) pesquisaram sobre o Facebook e a identidade social de adolescentes; Kross et al. (2013) estudaram a influência do Facebook na sensação de bem-estar dos usuários; Morris (2013) investigou a diferença de gêneros entre os usuários do Facebook; Ellison et al. (2014) conduziram uma pesquisa sobre os processos de capital social derivados dos relacionamentos da rede Facebook.

De acordo com Ellison et al (2014) pesquisas têm demonstrado a existência de uma relação entre o uso do Facebook e o aumento dos níveis de capital social. Os autores explicam que o capital social pode ser descrito como a capacidade dos indivíduos ou grupos acessarem recursos existentes nos círculos sociais destes. Para Ellison et al, o Facebook é uma rede social que se mostra bastante adequada para a construção do capital social. Pesquisa conduzida por Ellison et al (2014) teve por objetivo estudar a relação entre capital social e o engajamento dos usuários em um conjunto específico de comportamentos de comunicação. Eles avaliaram, por exemplo, a intenção dos usuários de aceitar novos amigos, bem como de estimular os vínculos já existentes por meio de mensagens de "Feliz Aniversário" ou respondendo a pedidos de ajuda ou indicações.

O presente artigo faz um recorte do estudo feito por Ellison et al (2014) para investigar as diferenças entre as interações sociais que acontecem no Facebook daquelas que ocorrem face a face. $\mathrm{O}$ entendimento desses processos é relevante para a área de Publicidade e Propaganda, pois pode auxiliar na compreensão de como as novas formas de tecnologia influenciam os relacionamentos entre os indivíduos. Barros (2010) lembra que as mídias digitais interativas mudaram a forma pela qual as pessoas interagem umas com as outras e com as empresas. Além disso, o fluxo de comunicação permanente que está sendo estabelecido na sociedade em rede e a multiplicação de conexões entre sistemas, pessoas e máquinas propiciam o relacionamento mercadológico. Outra relevância que o tema abordado neste artigo propicia consiste na oportunidade de replicar, no Brasil, um estudo científico conduzido originalmente com alunos de uma universidade norte-americana. A comparação entre os dois resultados de pesquisa pode trazer avanços para o conhecimento na área.

Assim, com base na proposta de replicação do estudo de Ellison et al (2014), este artigo trata do seguinte problema de pesquisa: "As interações sociais no Facebook promovem uma maior formação de capital social do tipo bridging do que as interações que ocorrem fora da rede?”. Ele teve por objetivo geral "analisar a formação de capital social do tipo bridging dentro e fora da rede Facebook". Dois objetivos específicos foram estabelecidos: 1) traduzir e validar, no Brasil, os questionários "General Bridging Social Capital" e "Facebook Bridging Social Capital", utilizados em um estudo de Ellison et al (2014); 2) comparar, usando a versão traduzida dos questionários de Ellison et al (2014), as interações que ocorrem no Facebook e no meio social na criação de capital social do tipo bridging.

Um breve referencial teórico dos principais conceitos envolvidos no tema pesquisado será apresentado a seguir. A descrição da metodologia de pesquisa utilizada no estudo e a apresentação e discussão dos resultados encontrados completam este artigo.

\section{Os Relacionamentos na Era Digital}

A tecnologia revolucionou profundamente a forma como as pessoas se relacionam. Bauman (2000) delimita o início da modernidade pela separação do tempo e espaço da prática de vida entre si, quando a distância percorrida numa unidade de tempo passou a depender da tecnologia e não mais de ferramentas naturais de mobilidade, havendo assim uma transformação nas redes de interatividade. As inovações nos meios de comunicação e tecnologia possibilitaram às pessoas se manterem conectadas via smartphone ou outros aparelhos dotados de internet durante todo o dia. $\mathrm{O}$ uso desses multicanais 
de comunicação para se manter conectado foi definido por Wellman (2001) como hiperconectividade. Essa hiperconectividade fez com que o foco da comunicação não mais fosse o conteúdo da mensagem a ser passada, mas sua circulação. Segundo Bauman (2003), manter o chat funcionando consiste no objetivo da comunicação em si, o silêncio ou a lentidão são punidos pela exclusão da conversa. O autor afirma que essa possibilidade de se aproximar e se afastar do outro com facilidade e rapidez promove simultaneamente o impulso de liberdade e a ânsia por pertencimento.

Observa-se, assim, que a busca constante e infindável por esse atributo permeia a sociedade moderna com um sentimento de insegurança e solidão, e o resultado é a necessidade de se comunicar, ainda que de maneira frívola e breve, com o maior número de indivíduos presentes em sua rede. Não é por acaso que se pode observar o sucesso das redes sociais e dos sites de relacionamento, aonde as relações virtuais, ainda que percebidas com uma menor importância do que as relações reais ou "off line", vigoram durante grande parte do dia das pessoas. A possibilidade de que em apenas alguns cliques essas relações possam gerar um impacto nas redes de relacionamento as transforma em verdadeiras fontes de incentivo, motivando o indivíduo a participar e a compartilhar conteúdo quase que ao mesmo tempo em que ele o experimenta.

Sites de redes sociais como o Facebook, segundo Luedtke (2003), penetram profundamente no dia a dia dos seus usuários e, por se tratar de uma tecnologia pervasiva, tendem a se tornar invisíveis uma vez que amplamente adotados. Esses sites, afirmam Tong, Kashian e Walther (2011), são plataformas ideais para que a manutenção e a reparação de relacionamentos ocorram de maneira rápida e com custo baixo, uma vez que possibilitam a disseminação ampla de mensagens e promovem a participação, feedback e interação por meio de múltiplos canais de comunicação.

Karjaluoto e Leppäniemi (2013) estudaram as razões que levam os usuários a participar de redes virtuais, atribuindo a elas um conjunto de determinações sociais que influenciam a intenção de se relacionar, como atitude, normas subjetivas, diversão, usabilidade e identidade social. Os autores observaram que um fator determinante para a participação nas redes é o sentimento de pertencimento e a percepção que o usuário tem da sua importância para a comunidade, apontando também que ferramentas de marketing como ads e pop-ups deveriam se valer desse conhecimento para sua eficácia, reforçando essas percepções no usuário.

\section{Capital social}

Matos (2009) afirma que a primeira análise sistemática do conceito de capital social surgiu em 1980, no artigo "Le capital social, notes provisoires" de Pierre Bourdieu. No artigo, o autor define capital social como o conjunto de recursos atuais e potenciais que estão ligados à posse de uma rede durável de relações institucionalizadas de interconhecimento e interreconhecimento. Bourdieu (1980) comenta que o capital social descreve circunstâncias nas quais os indivíduos podem se valer de sua participação em grupos e redes para atingir metas e benefícios. Segundo o autor, o capital social representa um conjunto de elementos com os quais uma classe social garante sua reprodução, justificando o uso do termo "capital" pelas suas características de acumulação (capital mobilizável), convertibilidade (capital humano transformado em capital social) e reciprocidade (indicadores de confiança).

Outra definição de capital social é a de Putnam et al (1993). Para esses autores, o capital social é entendido como um conjunto de características das organizações sociais de tal forma que são as redes, as normas partilhadas e a confiança, que facilitam a coordenação e a cooperação visando a um proveito mútuo. Putnam et al afirmam que essas redes, sustentadas pela colaboração de seus membros, constituem uma memória cultural que se torna fonte de orientação para ações futuras.

Putnam (2000) divide o capital social entre "bridging" e "bonding". Essas categorias descrevem recursos relacionados a diferentes tipos de relacionamento. Matos (2009) se refere a esta diferenciação como capital social de vinculação particularizada (bonding) e capital social de vinculação generalizada (bridging). Segundo Granovetter (1973), laços fracos que conectam diferentes partes de uma rede, como por exemplo amigo de um amigo, podem ser considerados laços do tipo bridging, e ajudam a propagar informações novas entre os grupos. Em oposição, o autor caracteriza os laços fortes pelas múltiplas interações e pelo alto índice de confiança, suporte e intimidade, promovendo acesso às formas mais objetivas de conversão de capital e associando-os ao capital social do tipo bonding como, por exemplo, um empréstimo financeiro. Haythornthwaite (2005) sugere que as pessoas usam múltiplos canais de 
comunicação para se comunicar com laços fortes e poucos canais para interagir com laços fracos.

Ainda sobre os tipos de capital social bridging e bonding, Warren (2001) os distingue pelas suas qualidades e benefícios. O autor explica que o capital social do tipo bonding cria uma forte lealdade dentro do grupo, incentivando a reciprocidade interna e a obtenção de recursos sociais e psicológicos. Em relação ao capital social do tipo bridging, afirma que este amplia as redes sociais e conecta grupos a recursos aos quais eles não teriam acesso de outro modo, aumentando o fluxo de informações que circula entre grupos de diferente vinculação e podendo gerar identidades coletivas e reciprocidade.

Bakshy et al (2012) afirmam que as redes no Facebook possuem os dois tipos de laços, fortes e fracos. Esses autores buscaram mensurar o comportamento de usuários do Facebook quando compartilhando informações. Durante o experimento, os autores estudaram a tendência de usuários em compartilhar um link que aparece no seu mural de notícias e fora dele. $\mathrm{O}$ resultado do experimento revelou que sujeitos expostos a sinalizações de amigos compartilhando conteúdo são altamente motivados a compartilhá-lo, e o fazem antes daqueles que não foram expostos. $\mathrm{O}$ estudo apontou ainda que os usuários são mais fortemente influenciados por laços fracos quando compartilhando links, pois ainda que relações do tipo bonding sejam individualmente mais influentes, o efeito de laços fortes não se equipara à grande abundância de laços fracos existentes na rede.

Ellison et al (2014) exploram o Facebook como uma plataforma que possibilita maior interação com os laços do tipo bridging, justificando o foco de seu estudo nesse tipo de capital por promover o acesso às informações novas. Os autores buscaram descobrir como o Facebook proporciona um maior acesso aos recursos inerentes aos laços fracos, estes que, diferente dos laços fortes, se dão de maneira mais difícil por outros canais. Ellison et al (2014) buscaram mensurar a percepção que os indivíduos têm de seus recursos dentro de dois ambientes sociais: o Facebook e o meio social como um todo. Para tal, os autores usaram a escala denominada 10 -item bridging scale criada por Williams (2006), que analisa aspectos como contato com outros, o sentimento de fazer parte de um grupo amplo, e interagir de maneira recíproca dentro de uma comunidade. A partir dessa escala, Ellison et al (2014) criaram mais uma de 10 itens para analisar as interações que ocorrem dentro da rede Facebook. O objetivo da pesquisa buscou capturar como as percepções de par- ticipantes podem variar quando pensando no meio social deles além de uma medida separada de capital social do tipo bridging que focava especificamente nos recursos associados a amigos do Facebook. Os autores sugerem um relacionamento entre o uso do Facebook e a percepção de capital social do tipo bridging, tomando-se por base que laços fracos tendem a ser laços do tipo bridging, o capital social do tipo bridging se beneficia do uso do Facebook por uma questão técnica do site, que diminui o custo de se cultivar e se comunicar com uma ampla rede de laços fracos. Além disso, o Facebook possibilitaria aos usuários entrarem em contato com informações relacionadas à identidade de outros indivíduos, permitindo também que haja uma troca de informações de maneira fácil e rápida, por meio de mensagens de textos, imagens e vídeos.

O estudo desenvolvido por Ellison et al (2014) sobre as interações que ocorrem em dois tipos de ambientes sociais serviu de base para a pesquisa descrita neste artigo.

\section{Método de pesquisa}

A pesquisa descrita neste trabalho foi do tipo exploratória e fez uso da abordagem quantitativa, tendo sido realizada no Centro Universitário de Brasília (UniCEUB), no campus da Asa Norte, envolvendo uma amostra formada por alunos de graduação. Ser usuário do Facebook foi o critério de inclusão para participar da pesquisa que envolveu 225 pessoas sendo que a amostra final ficou com 220 sujeitos, após serem descartados os questionários que foram preenchidos de forma incompleta ou incorreta. A análise das características demográficas indicou que 53,9\% dos participantes pertencia ao sexo masculino; $74,8 \%$ era formado por pessoas jovens, na faixa etária entre 20 a 30 anos de idade; 60,4\% dos respondentes trabalham; 63,8\% estuda no período diurno e $46,3 \%$ estão matriculados no curso de Publicidade e Propaganda.

$\mathrm{O}$ instrumento utilizado na pesquisa foi uma versão traduzida e adaptada dos dois questionários usados por Ellison et al (2014), o "General Bridging Social Capital" e o "Facebook Bridging Social Capital". Cada questionário possuia 10 itens para serem respondidos com base em uma escala do tipo "Likert", variando de " 1 = discordo totalmente" até " 5 = concordo totalmente"., tendo sido acrescido um campo para levantamento dos dados demográficos dos participantes da pesquisa. Foram impressas 225 cópias do instrumento. Para garantir a aleatoriedade da amostra, ele foi aplicado pelo próprio pesquisador, 
durante os intervalos das aulas dos períodos matutino e noturno. Os alunos foram abordados aleatoriamente e indagados se faziam uso do Facebook. Quando respondiam positivamente, eram convidados a participar da pesquisa.

Os dados foram analisados pelo Statistical Package for Social Sciences (SPSS). Foi feita uma análise exploratória para verificar a normalidade das distribuições e os pressupostos da análise fatorial. Em seguida, foram feitas análises fatoriais e de consistência interna dos itens que compunham cada fator, além de estatísticas descritivas, tais como média, desvio padrão e erro padrão das respostas dos sujeitos nos fatores extraídos. Usou-se um “teste-t” para verificar a diferença entre as médias dos dois questionários.

\section{Apresentação e discussão dos resultados}

Com relação ao primeiro objetivo da pesquisa, foi realizada a tradução do Inglês para o Português dos questionários "General Bridging Social Capital” e "Facebook Bridging Social Capital" utilizados no estudo de Ellison et al (2014). Na versão traduzida, buscando melhor atender aos fatores culturais do novo idioma e seguindo a terminologia da literatura científica da área, optou-se por consolidar os dois questionários em um único instrumento denominado de "Escala de Capital Social de Vinculação Generalizada”. Na primeira parte desse instrumento, estavam os 10 itens referentes ao questionário "Facebook Bridging Social Capital" que trata das interações entre o indivíduo e seus amigos do Facebook e, na segunda parte, os 10 itens do "General Bridging Social Capital" que trata das interações entre o indivíduo e seu meio social como um todo, incluindo, por exemplo, parentes, amigos, colegas etc. Assim, embora os dois questionários tenham sido agrupados em um único instrumento, a integridade individual de cada um deles foi preservada, uma vez que se trata de dois questionários distintos.

$\mathrm{O}$ instrumento traduzido foi aplicado em uma amostra de cinco voluntários, com o mesmo nível de escolaridade dos futuros participantes da pesquisa, para verificação da clareza do conteúdo dos itens (análise semântica). $\mathrm{O}$ resultado mostrou não haver necessidade de se fazer alteração em nenhum dos itens traduzidos. Descartados cinco questionários que tinham sido preenchidos de forma incompleta ou incorreta a amostra final ficou com um total de 220 sujeitos.

Os dados foram tabulados usando-se o Statistical Package for Social Sciences (SPSS). Após a tabulação, todas as análises estatísticas foram feitas separadamente para cada um dos dois questionários que compunham o instrumento "Escala de Capital Social de Vinculação Generalizada”. Assim, o banco de dados formado pelos 220 sujeitos foi submetido a uma análise preliminar para verificar se a entrada desses dados foi feita de forma correta, identificar os valores ausentes (missings), o ajustamento entre as distribuições das variáveis e os pressupostos exigidos para se efetuar análise multivariada. Em seguida, foram feitas análises fatoriais e de confiabilidade buscando a validação de cada um dos dois questionários.

Para a primeira parte da "Escala de Capital Social de Vinculação Generalizada”, que trata das interações entre os sujeitos da pesquisa e seus amigos no Facebook ("Facebook Bridging Social Capital"), os resultados da análise dos componentes principais apontaram que a medida de adequação amostral Kaiser-Meyer-Olkin (KMO) foi de 0,78 e o resultado para o teste de esfericidade de Bartlett apresentou uma probabilidade igual a $\mathrm{p}<0.000001$. A estrutura empírica indicada foi de até três fatores que explicavam cerca de 63,15\% da variância total das respostas dos sujeitos aos itens do questionário. Com essa indicação foi feita a análise fatorial do instrumento forçando para um, dois e três fatores pelo processo de extração "análise dos eixos principais" (PAF), utilizando-se a rotação "Varimax", a mesma utilizada por Ellison et al (2014) quando da validação dos seus questionários. Foram consideradas somente cargas fatoriais maiores que 0,35 . A solução mais adequada mostrou ser a de um único fator com uma variância explicada de 30,53\%. Não foi descartado item e foi dado ao fator o nome de "capital social de vinculação generalizada para o Facebook", com média de 3,52, desvio padrão de 0,74 e um alpha de Cronbach de 0,83:

Tabela 1 - Estrutura empírica do fator "Capital Social de Vinculação Generalizada para o Facebook"

\begin{tabular}{cc}
\hline Itens & Carga \\
\hline 1 & 0,61 \\
2 & 0,61 \\
3 & 0,47 \\
4 & 0,52 \\
5 & 0,57 \\
6 & 0,72 \\
7 & 0,48 \\
8 & 0,34 \\
9 & 0,58 \\
10 & 0,57 \\
\hline Eigenvalue & 3,72 \\
$\%$ Variância & 37,16 \\
Alpha de Cronbach & 0,81 \\
\hline
\end{tabular}

Fonte: Dados da Pesquisa 
Observa-se na Tabela 1 que o item 8, cuja descrição é "Faz com que eu queira usar parte do meu tempo para apoiar as atividades comunitárias no Facebook", possui a menor carga, evidenciando que ele possui uma baixa correlação com o fator. $\mathrm{O}$ item 6 , de maior carga, diz respeito à sensação de se conectar com uma visão de mundo maior. A comparação entre esses resultados indica que os usuários do Facebook, ainda que possuam noção dos diferentes graus de vinculação existentes na rede, não têm interesse em participar de atividades que possam fazê-los entrar em contato com pessoas com quem eles possuem laços mais fracos. A segunda menor carga foi a do item 3, cuja descrição "Faz com que eu me interesse sobre o que pessoas diferentes de mim estão pensando", indica mais uma vez a falta de interesse dos usuários em se relacionar com laços mais fracos da sua rede.

Para a segunda parte da "Escala de Capital Social de Vinculação Generalizada", que trata das interações entre os sujeitos da pesquisa e seu meio social mais abrangente (parentes, amigos, colegas etc.), também foram feitas as mesmas análises descritas anteriormente para a primeira parte do instrumento, sendo que o índice KMO obtido foi de 0,79 e o teste de esfericidade de Bartlett apresentou uma probabilidade igual a $\mathrm{p}<0,000001$. Na estimativa, quanto ao número de fatores possíveis, a análise dos componentes principais indicou uma estrutura empírica de até três fatores que explicavam cerca de $62,7 \%$ da variância total das respostas dos sujeitos aos itens do questionário. Foi feita a análise fatorial forçando para um, dois e três fatores pelo processo de extração "análise dos eixos principais" (PAF), utilizando-se a rotação "Varimax". Foram consideradas somente cargas fatoriais maiores que 0,35. A exemplo do outro questionário, a solução mais adequada mostrou ser a de um fator com uma variância explicada de $32,76 \%$. Não foi descartado item e o fator foi chamado de "capital social de vinculação generalizada”, com média de 3,58, desvio padrão de 0,74 e um alpha de Cronbach de 0,81 :

Tabela 2 - Estrutura empírica do fator "Capital Social de Vinculação Generalizada"

\begin{tabular}{cl} 
Itens & Carga \\
\hline 1 & 0,53 \\
2 & 0,63 \\
3 & 0,64 \\
4 & 0,44 \\
5 & 0,42 \\
6 & 0,69 \\
7 & 0,57 \\
8 & 0,59 \\
9 & 0,50 \\
10 & 0,56 \\
\hline Eigenvalue & 3,93 \\
\% Variância & 39,32 \\
Alpha de Cronbach & 0,83 \\
\hline
\end{tabular}

Observa-se na Tabela 2 que as cargas dos itens do fator "Capital Social de Vinculação Generalizada" são mais homogêneas do que aquelas que estruturam o fator "Capital Social de Vinculação Generalizada para o Facebook". Isso indica que, para a amostra em questão, há maior facilidade de se avaliar a construção do capital social do tipo bridging no meio social do que na plataforma Facebook. Uma das razões que pode ter levado a essa facilidade atribui-se ao fato de que as interações sociais que ocorrem no mundo real são comumente de caráter comunitário, como por exemplo a prática de esportes, os relacionamentos entre grupos dentro da sala de aula, no ambiente de trabalho e nas festas. Quando se trata das interações sociais dentro do Facebook, essas acontecem normalmente de indivíduo para indivíduo, como por exemplo as ações de "curtir" ou comentar uma foto de um amigo, ou o envio de mensagens privadas. Ainda que a formação do capital social do tipo bridging aconteça quando existem interações com laços mais fracos, como por exemplo interações sociais com amigos de amigos, elas são tipicamente mediadas por sujeitos de laços mais fortes. Atribui-se assim a importância de se relacionar com grupos de laços fortes para se conseguir acessar recursos de laços mais fracos.

Outro fato que corrobora a razão citada acima para a facilidade dos sujeitos em avaliar a construção de capital social do tipo bridging no meio social refere-se ao aumento substancial da carga relacionada ao item "Faz com que eu queira usar parte do meu tempo para apoiar as atividades comunitárias do meu meio social" do fator "Capital Social de Vinculação Generalizada" (item 4), quando comparada à carga do mesmo item no fator "Capital Social de Vinculação Generalizada para o Facebook" (item 8). Uma vez que atividades comunitárias propiciam um ambiente de convergência de laços fracos, a baixa correlação desse item com o fator relacionado ao Facebook indica que as interações sociais que lá ocorrem são de caráter mais individualizado, estas que são mais normalmente atribuídas à manutenção de relacionamentos preexistentes do que a construção de novos laços (LENHART, 2009).

Os resultados obtidos com a validação dos dois questionários na versão em Português são muito parecidos com os resultados encontrados no estudo de Ellison et al (2014). Em ambos os estudos, as médias dos dois questionários ficaram entre os pontos 3 e 4 da escala, os desvios padrões foram baixos e os Alphas de Cronbach 
altos. Esses resultados parecem confirmar a boa estrutura desse instrumento de pesquisa para se investigar as relações que ocorrem na formação de capital social do tipo bridging dentro e fora do Facebook.

Com relação ao segundo objetivo específico, comparar os dois tipos de interação na criação de capital social do tipo bridging, foi realizado um "teste-t" dependente com os fatores "capital social de vinculação generalizada" e "capital social de vinculação generalizada para o Facebook" com o objetivo de verificar se as médias encontradas eram ou não significativamente diferentes. A realização desse teste buscou examinar se o capital social gerado pela vinculação generalizada para o Facebook era significativamente maior que o capital social gerado pela vinculação generalizada para o meio social.

$\mathrm{O}$ resultado mostrou que as duas médias não são significativamente diferentes $\mathrm{t}(219)=-1,12, \mathrm{p}=0,262$. Isso sugere que o Facebook, ainda que seja um meio que possibilite aos usuários construir capital social do tipo bridging mais facilmente, segundo Elllison et al (2014), não promove a criação desse tipo de capital de forma mais eficaz do que as interações no meio social fora dessa rede. Uma possível explicação para este resultado pode estar relacionada com o motivo pelo qual as pessoas fazem parte do Facebook. Segundo Lenhart (2009), a razão mais frequentemente citada para que indivíduos façam parte da rede é o cultivo de relacionamentos offline, ou seja, com pessoas que já possuem laços fortes com esses indivíduos. Ellison et al (2014) observaram que os usuários do Facebook conseguem diferenciar amigos "de verdade" de outros indivíduos adicionados, o que significa que os usuários têm consciência dos diferentes graus de vinculação que existem entre eles e os seus amigos na rede. Os autores evidenciaram ainda que, quanto menor a quantidade de amigos "de verdade" que os usuários possuíam na rede, maior é a construção de capital social do tipo bridging se comparando com pessoas com mais amigos "de verdade". Logo, o resultado encontrado pode ser reflexo de uma amostra que possui uma grande quantidade de amigos "de verdade" na rede Facebook, e que faz uso dela para cultivar os relacionamentos já existentes com laços fortes fora do Facebook.

\section{Conclusão}

Para o primeiro objetivo específico desta pesquisa os resultados obtidos com a aplicação dos dois questio- nários na versão em Português, mostraram-se bastante semelhantes aos resultados encontrados no estudo de Ellison et al (2014), sinalizando a boa estrutura desse instrumento de pesquisa para se investigar as relações que ocorrem na formação de capital social do tipo bridging dentro e fora do Facebook. Quanto ao segundo objetivo específico, o resultado encontrado nas comparações evidenciou que a criação do capital social do tipo bridging não é fortemente afetada pela utilização da rede Facebook, uma vez que não houve diferenças significativas quando comparando a criação desse tipo de capital social dentro e fora do Facebook.

Existem inúmeras limitações no presente estudo que podem enunciar resultados mais definitivos e talvez até mesmo contrários àqueles descobertos. Uma das limitações é que a pesquisa utiliza da percepção dos usuários para tentar mensurar a formação de capital social. Não existe na literatura atual um instrumento validado que demonstre de maneira determinante a formação do tipo de capital social estudado. Outra limitação refere-se ao fato de que a amostra estudada pode não refletir com exatidão o comportamento desse tipo de capital, tanto no Brasil quanto no mundo. Estudos futuros na área são imprescindíveis para que se possa atingir um nível de conhecimento que possibilite um entendimento mais amplo das relações dentro e fora das redes sociais.

O estudo contribuiu para o entendimento das relações que ocorrem entre os indivíduos dentro de redes sociais, apontando benefícios gerados por elas que ultrapassam a simples conexão entre pessoas, uma vez que a geração de capital social com laços mais fracos pode ser uma das consequências dos relacionamentos mediados por plataformas online. Uma outra contribuição do estudo foi a tradução e validação para o Português dos questionários "General Bridging Social Capital" e "Facebook Bridging Social Capital", utilizados por Ellison et al (2014). A realização de outras pesquisas com a utilização da versão traduzida dos questionários é necessária para a confirmação dos resultados obtidos neste estudo.

\section{Referências}

BAKSHY, Eytan et al. The role of social networks in information diffusion. In: INTERNATIONAL CONFERENCE ON WORLD WIDE WEB, 21., 2012, Lyon. Proceedings... Ann Arbor: Universidade of Michigan, 2012. p. 519-528. 
BARROS, Ana Cirne Paes de. Relacionamento mercadológico nas mídias interativas digitais: inovações na comunicação transmidiática entre empresas e consumidores. Conexões Midiaticas: Revista dos alunos do Programa de Pós-Graduação em Comunicação da UFPB, João Pessoa, n. 3, mar./ago. 2010. Disponível em: <http://www.academia.edu/3192349/ Relacionamento_Mercadol\%C3\%B3gico_nas_M\%C3\%ADdias_Interativas_Digitais_Inova\%C3\%A7\%C3\%B5es_na_ comunica\%C3\%A7\%C3\%A3o_transmidi\%C3\%A1tica_entre_empresas_e_consumidores $>$. Acesso em: 19 jun. 2015.

BAUMAN, Zygmunt. Modernidade líquida. Rio de Janeiro: J. Zahar, 2000.

BAUMAN, Zygmunt. Amor líquido. Rio de Janeiro: J. Zahar, 2003.

BOURDIEU, Pierre. Le capital social: notes provisoires. Actes de la Recherche en Sciences Sociales, Paris, v. 31, p. 2-3, jan. 1980.

CIRIBELI, João Paulo; PAIVA, Victor Hugo. Redes e mídias sociais na internet: realidades e perspectivas de um mundo conectado. Mediação, Belo Horizonte, v. 13, n. 12, p. 57-74, jan./jun. de 2011.

COLEMAN, James S. Social capital in the creation of human capital. American Journal of Sociology, Chicago, v. 94, p. S95-S120, 1988. Supplement: Organizations and institutions: sociological and economic approaches to the analysis of social structure.

CRUZ, Breno de Paula Andrade et al. Influência de brasileiros famosos no boicote de consumidores que usam redes sociais virtuais. Revista de Negócios, Natal, v. 17, n. 3, p. 91-110, jul./set. 2012.

ELLISON, Nicole B. et al. Cultivating social resources on social network sites: facebook relationship maintenance behaviors and their role in social capital processes. Journal of Computer-Mediated Communication, Pennsylvania, v. 19, n. 4, p. 855-870, July, 2014.

ELLISON, Nicole B.; STEINFIELD, Charles; LAMPE, Cliff. Connection strategies: social capital implications of facebook-enabled communication practices. New Media \& Society, Chicago, v. 13, n. 6, p. 45-62, Jun. 2011.

GRANOVETTER, Mark S. The strength of weak ties. American Journal of Sociology, Chicago, v. 78, n. 6, p. 1360-180, May, 1973.

HAYTHORNTHWAITE, Caroline. Social networks and Internet connectivity effects. Information, Communication \& Society, Florida, v. 8, n. 2, p. 125-147, Jun. 2005.

KARJALUOTO, Heikki; LEPPÄNIEMI, Matti. Social identity for teenagers: understanding behavioral intention to participate in virtual world environment. Journal of Theoretical and Applied Electronic Commerce Research, Talca, v. 8, n. 1, p. 1-16, Apr. 2013.
KROSS, Ethan et al. Facebook use predicts declines in subjective well-being in young adults. PLOS ONE. Strasbourg, v. 8, n. 8, p. 1-6, Aug. 2013.

LENHART, Amanda. Adults and social network websites. 2009. Disponível em: <http://www.pewinternet.org/files/ old-media/Files/Reports/2009/PIP_Adult_social_networking_data_memo_FINAL.pdf.pdf $>$. Acesso em: 19 jun. 2015.

LUEDTKE, Joe. Toward pervasive computing: in search of a unified communication model. 2003. Disponível em: <http://www.information-management.com/infodirect/20030418/6617-1.html>. Acesso em: 27 out. 2014.

MATOS, Heloiza. Capital social e comunicação: interfaces e articulações. São Paulo: Summus, 2009.

MORRIS, Erin. She “likes” it, he doesn't: gender differences in facebook communication behaviors. 2012. $42 \mathrm{f}$. Thesis (Graduation) - Department of Communication , University of Colorado at Boulder. Boulder: Undergraduate Honors Theses, Spring 2013.

PRIMO, Alex. Avaliação qualitativa de interações em redes sociais: relacionamentos no blog Martelada. Comunicação, Mídia e Consumo. São Paulo, v. 4, n. 11, p. 137-158, nov. 2007.

PUTNAM, Robert D. Bowling alone: the collapse and revival of American community. New York: Simon \& Schuster, 2000.

PUTNAM, Robert D. The prosperous community: social capital and public life. The american prospect, Washington, D.C., v. 4, n. 13, p. 35-42, Mar. 1993.

TONG, Stephanie Tom; WALTHER, Joseph B. Relational maintenance and CMC. In: WRIGHT, Kevin B.; WEBB, Lynne M. (Ed.). Computer-mediated communication in personal relationships. New York: P. Lang, 2011. p. 98-118.

WARREN, Mark E. Democracy and association. Princeton: Princeton University Press, 2001.

WELLMAN, Barry. Physical place and cyber place: the rise of networked individualism. International Journal of Urban and Regional Research, New Jersey, v. 25, n. 2, p. 227-252, Jun. 2001.

WILLIAMS, Dmitri. On and off the net: scales for social capital in an online era. Journal of Computer-Mediated Communication, Pennsylvania, v. 11, n. 2, p. 593-628, Jan. 2006. 